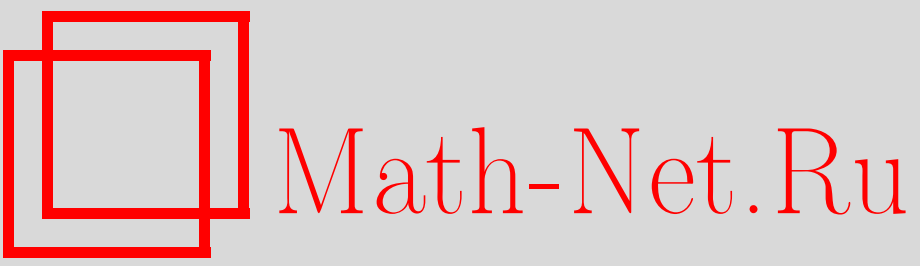

Ю. А. Фарков, Конечные фреймы Парсеваля в анализе Уолша, Итоги науки и техн. Сер. Соврем. мат. и ее прил. Темат. обз., 2019, том 170, 118-128

DOI: https://doi.org/10.36535/0233-6723-2019-170-118-128

Использование Общероссийского математического портала Math-Net.Ru подразумевает, что вы прочитали и согласны с пользовательским соглашением

http://www.mathnet.ru/rus/agreement

Параметры загрузки:

IP : 54.196 .121 .252

26 апреля 2023 г., 17:17:50 


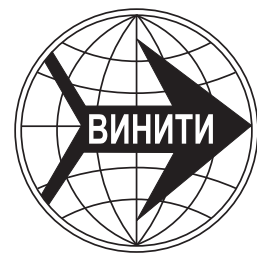

ИТОГИ НАУКИ И ТЕХНИКИ.

Современная математика и ее приложения.

Тематические обзоры.

Том 170 (2019). С. 118-128

DOI: $10.36535 / 0233-6723-2019-170-118-128$

УДК 517.518, 519.614

\title{
КОНЕЧНЫЕ ФРЕЙМЫ ПАРСЕВАЛЯ В АНАЛИЗЕ УОЛША
}

\author{
(C) 2019 г. $\quad$ Ю. A. ФАРКОВ
}

\begin{abstract}
АннотАция. Изложены методы построения конечных фреймов Парсеваля с помощью матриц Уолша и дискретного преобразования Виленкина-Крестенсона.

Ключевые слова: функция Уолша, фрейм, ортогональный всплеск, дискретное преобразование, проблема Кадисона-Зингера.
\end{abstract}

\section{FINITE PARSEVAL FRAMES IN WALSH ANALYSIS}

\section{(C) 2019 YU. A. FARKOV}

\begin{abstract}
Methods for constructing finite Parseval frames by using Walsh matrices and the discrete Vilenkin-Chrestenson transform are described.
\end{abstract}

Keywords and phrases: Walsh function, frame, orthogonal wavelet, discrete transformation, Kadison-Singer problem.

AMS Subject Classification: 42C15, 42C40

1. Введение. Теория конечных фреймов активно развивалась последние пятнадцать лет (см. библиографию в [34]) прежде всего в связи с приложениями в таких областях, как обработка сигналов, квантовая теория информации, многомерные ортогональные полиномы и сплайны, а также в теории сжатых измерений (compressed sensing). Основополагающие факты теории фреймов и её приложений содержатся в монографиях $[5,7,9,20]$, а некоторые недавние результаты о многомерных всплесковых фреймах изложены в [28]. Фреймы активно применяются для анализа полноты, устойчивости и избыточности дискретных представлений сигналов. Понятие фрейма Парсеваля обобщает понятие ортонормированного базиса на системы, не обладающие свойством минимальности (см., например, $[9, \S 1.8]$ ). В настоящей статье излагаются методы построения конечных фреймов Парсеваля с помощью функций Уолша и дискретного преобразования Виленкина-Крестенсона.

Хорошо известно, что функции Уолша можно интерпретировать как характеры двоичной группы Кантора, а обобщениями функций Уолша являются характеры групп Виленкина (см., например, $[1,4,30,32,32])$. Локально компактная канторова группа $\mathcal{C}$ может быть определена как аддитивная группа диадического поля $\mathbb{F}$. Первые примеры ортогональных всплесков на группе $\mathcal{C}$ были найдены в [29], а полученные к настоящему времени результаты о всплесках на группах Кантора и Виленкина изложены в книге [25] и обзорных статьях $[14,22]$. В [21] фреймы на группе $\mathcal{C}$ были определены с помощью допустимого условия Добеши и обобщенных ядер Дирихле. Кратномасштабный анализ для построения фреймов Парсеваля на группах Виленкина применялся в [15,22-24]. В разделе 2 настоящей статьи приведены используемые в дальнейшем обозначения и краткие сведения о дискретных функциях Уолша. В разделе 3 показано, как для построения фреймов Парсеваля применяются матрицы Уолша. В связи с проблемой Кадисона-Зингера приведен недавний результат [16] о расщеплении конечных фреймов Парсеваля. В заключительном 
разделе 4 доказано обобщение теоремы о фреймах в пространствах периодических последовательностей, сформулированной в [10], и приведен алгоритм построения фреймов Парсеваля с помощью дискретного преобразования Виленкина-Крестенсона.

2. Дискретные функции Уолша. Как обычно, через $\mathbb{Z}_{+}$и $\mathbb{N}$ обозначаются множества целых неотрицательных и натуральных чисел соответственно. Система Уолша $\left\{w_{l}: l \in \mathbb{Z}_{+}\right\}$является ортонормированным базисом в $L^{2}[0,1]$ и определяется следующим образом (см. $\left.[4,30]\right)$. Пусть функция $r_{0}(x)$ имеет период 1 и задана на интервале $[0,1)$ по формуле

$$
r_{0}(x)=\left\{\begin{aligned}
1, & x \in[0,1 / 2), \\
-1, & x \in[1 / 2,1) .
\end{aligned}\right.
$$

Тогда

$$
w_{0}(x) \equiv 1, \quad w_{l}(x)=\prod_{j=1}^{k}\left(r_{0}\left(2^{j-1} x\right)\right)^{\nu_{j}}, \quad l \in \mathbb{N}, \quad x \in[0,1],
$$

где $\nu_{j}$ - цифры двоичного разложения числа $l$ :

$$
l=\sum_{j=1}^{k} \nu_{j} 2^{j-1}, \quad \nu_{j} \in\{0,1\}, \quad \nu_{k}=1, \quad k=k(l) .
$$

При $0 \leqslant l \leqslant 2^{n}-1$ функции Уолша $w_{l}(x)$ постоянны на двоичных интервалах $\left[k / 2^{n},(k+1) / 2^{n}\right)$, а значения $w_{l k}=w_{l}\left(k / 2^{n}\right)$ задают матрицу Уолша $W_{n}=\left[w_{l k}\right]_{l, k=0}^{n}$. В частности, имеем

$$
W_{1}=\left[\begin{array}{rr}
1 & 1 \\
1 & -1
\end{array}\right], \quad W_{2}=\left[\begin{array}{rrrr}
1 & 1 & 1 & 1 \\
1 & 1 & -1 & -1 \\
1 & -1 & 1 & -1 \\
1 & -1 & -1 & 1
\end{array}\right] \text {. }
$$

При любом натуральном $n$ матрица $W_{n}$ симметрична и удовлетворяет соотношениям ортогональности

$$
\sum_{j=0}^{2^{n}-1} w_{l j} w_{k j}=\sum_{i=0}^{2^{n}-1} w_{i l} w_{i k}=2^{n} \delta_{l k}, \quad l, k \in\left\{0,1, \ldots, 2^{n}-1\right\},
$$

где $\delta_{l k}$ - символ Кронекера. Строки матриц $W_{n}$ называют дискретными функииями Уолша, упорядоченными по Пэли (см., например, [8, гл. 2]).

На практике часто используют функции Уолша, упорядоченные по Адамару (см., например, $[6,8])$. По определению $H_{1}:=W_{1}$, а матрица $H_{n}=\left[h_{l k}\right]_{l, k=0}^{2^{n}-1}$ совпадает с $n$-й кронекеровой степенью матрицы $H_{1}$. Например, для $n=2$ имеем

$$
H_{2}=H_{1} \otimes H_{1}=\left[\begin{array}{rrrr}
1 & 1 & 1 & 1 \\
1 & -1 & 1 & -1 \\
1 & 1 & -1 & -1 \\
1 & -1 & -1 & 1
\end{array}\right] .
$$

Используемый в MATLAB рекурсивный метод Сильвестра генерирования матриц $H_{n}$ основан на тождестве $H_{n+1}=H_{1} \otimes H_{n}$. Матрицы $H_{n}$ симметричны, обладают свойством ортогональности вида (1) и служат основным примером матриц Адамара, имеющих многочисленные применения в обработке сигналов (см. [8, гл. 2], [2,26,31]). Более 100 лет остается не доказанной гипотеза о существовании для любого натурального $m$ матрицы Адамара порядка $4 m$ (см., например, недавнюю статью [17]). Отметим также, что если известны цифры двоичных разложений

$$
l=\sum_{j=1}^{n} l_{j} 2^{j-1}, \quad k=\sum_{j=1}^{n} k_{j} 2^{j-1}, \quad l_{j}, k_{j} \in\{0,1\},
$$

то элементы матриц $W_{n}$ и $H_{n}$ вычисляются по формулам

$$
w_{l k}=(-1)^{w(l, k)}, \quad h_{l k}=(-1)^{h(l, k)},
$$


где

$$
w(l, k)=\sum_{j=1}^{n} l_{j} k_{n-j+1}, \quad h(l, k)=\sum_{j=1}^{n} l_{j} k_{j}, \quad l, k \in\left\{0,1, \ldots, 2^{n}-1\right\} .
$$

Используемые в разделе 4 обобщения функций Уолша называют функииями Крестенсона или функциями Виленкина-Крестенсона (см. исторические сведения и библиографию в $[2,4,6])$.

3. Фреймы Парсеваля, определяемые по матрицам Уолша. В [3] найдены ортонормированные базисы в собственных подпространствах дискретного преобразования Уолша и построены соответствующие этим базисам фреймы Парсеваля. В дополнение к этому результату обсудим взаимосвязи между конечными фреймами Парсеваля и матрицами Уолша. Хорошо известна следующая характеристика фреймов Парсеваля для пространства $\mathbb{C}^{d}$ произвольной размерности $d$.

Предложение 3.1. Пусть $\Lambda$-матрица размера $d \times s$, столбцами которой являются векторы $\mathbf{v}_{1}, \mathbf{v}_{2}, \ldots, \mathbf{v}_{s}$ пространства $\mathbb{C}^{d}$. Следующие утверждения эквивалентны:

(i) $\Lambda \Lambda^{*}=I_{d}$, где $I_{d}-$ единичная матрича порядка $d$;

(ii) строки матрицы $\Lambda$ образуют ортонормированную систему в $\mathbb{C}^{s}$;

(iii) векторы $\mathbf{v}_{1}, \mathbf{v}_{2}, \ldots, \mathbf{v}_{s}$ образуют фрейм Парсеваля для $\mathbb{C}^{d}$.

Пусть $N=2^{n}$, где $n$-натуральное число. Напомним, что векторы $\mathbf{v}_{1}, \mathbf{v}_{2}, \ldots, \mathbf{v}_{s}$ образуют фрейм Парсеваля для $\mathbb{C}^{N}$, если для каждого $\mathbf{x} \in \mathbb{C}^{N}$

$$
\|\mathbf{x}\|^{2}=\sum_{k=1}^{s}\left|\left\langle\mathbf{x}, \mathbf{v}_{k}\right\rangle\right|^{2}
$$

или, эквивалентно,

$$
\mathbf{x}=\sum_{k=1}^{s}\left\langle\mathbf{x}, \mathbf{v}_{k}\right\rangle \mathbf{v}_{k}
$$

Здесь $s \geqslant N$, причем в случае $s=N$ фрейм $\left\{\mathbf{v}_{1}, \mathbf{v}_{2}, \ldots, \mathbf{v}_{s}\right\}$ является ортонормированным базисом в $\mathbb{C}^{N}$. Для произвольной ортогональной матрицы $G$ размера $N \times N$ выберем $m<N$, запишем

$$
G=\left[\mathbf{g}_{1}, \ldots, \mathbf{g}_{m} \mid \mathbf{g}_{m+1}, \ldots, \mathbf{g}_{N}\right]
$$

и определим матрицу $G_{1} \in \mathbb{R}^{N \times m}$ равенством $G_{1}=\left[\mathbf{g}_{1}, \ldots, \mathbf{g}_{m}\right]$. Обозначим через $V=\left[\mathbf{v}_{1}, \ldots, \mathbf{v}_{N}\right]$ матрицу, сопряжённую к $G_{1}$. Согласно предложению 3.1 векторы $\mathbf{v}_{1}, \ldots, \mathbf{v}_{N}$ образуют фрейм Парсеваля для $\mathbb{C}^{m}$. В [16] эта конструкция применяется к нормализованной матрице Уолша $H_{n} / \sqrt{N}$, а фрейм Парсеваля, определённый столбцами полученной таким способом матрицы

$$
V=\left[\mathbf{v}_{1}, \ldots, \mathbf{v}_{N}\right] \in \mathbb{R}^{m \times N},
$$

называется фреймом Уолша. Легко видеть, что $\left\|\mathbf{v}_{j}\right\|=\sqrt{m / N}$ для всех $j \in\{1, \ldots, N\}$.

Что произойдет, если попытаться расщепить фрейм Уолша на два равных фрейма? Этот вопрос имеет отношение к известной проблеме Кадисона-Зингера. В 1959 г. эта проблема была сформулирована следующим образом (см. [27]): «Does every pure state on the (abelian) von Neumann algebra $D$ of bounded diagonal operators on the Hilbert space $\ell^{2}$ have a unique extension to a state on $B\left(\ell^{2}\right)$, the von Neumann algebra of all bounded linear operators on $\ell^{2}$ ?» Проблема КадисонаЗингера решена в 2013 г. и эквивалентна ряду других известных задач: о базисах гильбертова пространства, о замощении бесконечных матриц, о разбиениях фреймов, об обратимости конечных матриц с доминирующей диагональю, о тригонометрических суммах на канторовых множествах, о комбинаторных свойствах систем векторов в $\mathbb{R}^{d}$ (см., например, $\left.[19,33]\right)$. Основные результаты о конечномерных интерпретациях проблемы Кадисона-Зингера анализируются в [18] (см. также [12]). Следующее предложение было сформулировано в [35]; оно иногда называется «утверждением WDS» (weaver discrepancy statement).

Предложение 3.2. Пусть векторь $\mathbf{v}_{1}, \mathbf{v}_{2}, \ldots, \mathbf{v}_{s}$ образуют фрейм Парсеваля для $\mathbb{C}^{d}$. Предположим, что $\left\|\mathbf{v}_{j}\right\|^{2} \leqslant \alpha$ для всех $j \in\{1, \ldots, s\}$. Тогда существует такое разбиение множества 
индексов $J=\{1, \ldots, s\}$ на два непересекающихся подмножества $J_{1}$ и $J_{2}$, что для всех $\mathbf{x} \in \mathbb{C}^{d}$, $\|\mathbf{x}\|=1$ выполняются неравенства

$$
\left.\left|\sum_{j \in J_{k}}\right|\left\langle\mathbf{x}, \mathbf{v}_{j}\right\rangle\right|^{2}-\frac{1}{2} \mid \leqslant 5 \sqrt{\alpha}, \quad k=1,2 .
$$

Из равенства

$$
H_{n}=\left[\begin{array}{rr}
H_{n-1} & H_{n-1} \\
H_{n-1} & -H_{n-1}
\end{array}\right]
$$

видно, что при $m \leqslant N / 2$ фрейм Парсеваля $\left\{\mathbf{v}_{1}, \ldots, \mathbf{v}_{N}\right\}$ для $\mathbb{C}^{m}$, заданный матрицей (3), разбивается на два одинаковых жёстких фрейма для $\mathbb{C}^{m}$, соответствующим матрицам

$$
V_{1}=\left[\mathbf{v}_{1}, \ldots, \mathbf{v}_{N / 2}\right], \quad V_{2}=\left[\mathbf{v}_{N / 2+1}, \ldots, \mathbf{v}_{N}\right],
$$

причем $V_{1} V_{1}^{*}=V_{2} V_{2}^{*}=I_{m} / 2$.

Пример 3.1. Пусть $m=3$ и $N=8$. Тогда изложенная выше конструкция приводит к матрице

$$
V=\frac{1}{2 \sqrt{2}}\left[\begin{array}{rrrr|rrrr}
1 & 1 & 1 & 1 & 1 & 1 & 1 & 1 \\
1 & -1 & -1 & 1 & 1 & -1 & -1 & 1 \\
1 & 1 & -1 & -1 & 1 & 1 & -1 & -1
\end{array}\right]=\left[V_{a} \mid V_{b}\right]
$$

для которой $V V^{*}=I_{3}$. Определяемый этой матрицей фрейм Парсеваля расщепляется на два одинаковых фрейма, причем $V_{a} V_{a}^{*}=V_{b} V_{b}^{*}=I_{3} / 2$. На следующем шаге после изменения нормировки получается матрица

$$
V=\frac{1}{2}\left[\begin{array}{rr|rr}
1 & 1 & 1 & 1 \\
1 & -1 & -1 & 1 \\
1 & 1 & -1 & -1
\end{array}\right]=\left[V_{a} \mid V_{b}\right]
$$

для которой $V V^{*}=I_{3}$. Однако теперь $V_{a} \neq V_{b}$ и расщепление на одинаковые фреймы невозможно. При этом

$$
\left\|V_{a} V_{a}^{*}-I_{3} / 2\right\|_{2}=\left\|V_{b} V_{b}^{*}-I_{3} / 2\right\|_{2}=1 / 2
$$

где $\|\cdot\|_{2}-$ спектральная матричная норма.

Основной результат статьи [16] относится к случаю $m>N / 2$ и формулируется следующим образом.

Теорема 3.1. Пусть $N / 2<m<N, s=m-N / 2$, где $m \in \mathbb{N}$. Предположим, что матрица $Y \in \mathbb{R}^{N \times m}$ получена удалением из правой части матрицы $H_{n}$ произвольно выбранных $N / 2-s$ столбиов, так что

$$
Y=\left[\begin{array}{rrr|rrr}
\mathbf{y}_{1} & \ldots & \mathbf{y}_{N / 2} & \mathbf{y}_{k(1)} & \cdots & \mathbf{y}_{k(s)} \\
\hline \mathbf{y}_{1} & \cdots & \mathbf{y}_{N / 2} & -\mathbf{y}_{k(1)} & \cdots & -\mathbf{y}_{k(s)}
\end{array}\right]=\left[\begin{array}{c}
Y_{a} \\
\hline Y_{b}
\end{array}\right]
$$

где $1 \leqslant k(1)<\cdots<k(s) \leqslant N / 2$. Положим

$$
V=\frac{Y^{*}}{\sqrt{N}}, \quad V_{a}=\frac{Y_{a}^{*}}{\sqrt{N}}, \quad V_{b}=\frac{Y_{b}^{*}}{\sqrt{N}} .
$$

Тогда $V V^{*}=I_{N}, V=\left[V_{a} \mid V_{b}\right]$ и вернь равенства

$$
V_{a} V_{a}^{*}-I_{N / 2} / 2=\left[\begin{array}{c|c}
0 & \Delta / 2 \\
\hline \Delta^{*} / 2 & 0
\end{array}\right], \quad V_{b} V_{b}^{*}-I_{N / 2} / 2=\left[\begin{array}{c|c}
0 & -\Delta / 2 \\
\hline-\Delta^{*} / 2 & 0
\end{array}\right],
$$

где $\Delta^{*} \Delta=I_{s}, \Delta \in \mathbb{R}^{(N / 2) \times s}$. Более того,

$$
\left\|V_{a} V_{a}^{*}\right\|_{2}=\left\|V_{b} V_{b}^{*}\right\|_{2}=1
$$

и для погрешностей верны равенства

$$
\begin{gathered}
\left\|V_{a} V_{a}^{*}-I_{N / 2} / 2\right\|_{2}=\left\|V_{b} V_{b}^{*}-I_{N / 2} / 2\right\|_{2}=1 / 2, \\
\left\|V_{a} V_{a}^{*}-I_{N / 2} / 2\right\|_{F}=\left\|V_{b} V_{b}^{*}-I_{N / 2} / 2\right\|_{F}=\sqrt{(m-N / 2) / 2}
\end{gathered}
$$

где $\|\cdot\|_{F}-$ норма Фробениуса. 
Пример 3.2. Пусть $N=8$ и $m=6$ (тогда, очевидно, $s=2$ ). Если выбрать $k(1)=1$ и $k(2)=3$, то матрица $Y$ примет вид

$$
Y=\left[\begin{array}{llll|rr}
\mathbf{y}_{1} & \mathbf{y}_{2} & \mathbf{y}_{3} & \mathbf{y}_{4} & \mathbf{y}_{1} & \mathbf{y}_{3} \\
\hline \mathbf{y}_{1} & \mathbf{y}_{2} & \mathbf{y}_{3} & \mathbf{y}_{4} & -\mathbf{y}_{1} & -\mathbf{y}_{3}
\end{array}\right]
$$

В этом случае

$$
\Delta=\left[\begin{array}{ll}
1 & 0 \\
0 & 0 \\
0 & 1 \\
0 & 0
\end{array}\right], \quad \Delta \Delta^{*}=\left[\begin{array}{llll}
1 & 0 & 0 & 0 \\
0 & 0 & 0 & 0 \\
0 & 0 & 1 & 0 \\
0 & 0 & 0 & 0
\end{array}\right], \quad\left\|V_{a} V_{a}^{*}-I_{4} / 2\right\|_{F}=\left\|V_{b} V_{b}^{*}-I_{4} / 2\right\|_{F}=1
$$

что согласуется с теоремой 3.1.

В [16] приведены примеры, проясняющие в терминах квадратичных форм связь теоремы 3.1 с проблемой Кадисона-Зингера.

Покажем, что любой конечный фрейм Парсеаваля, составленный из векторов одинаковой длины, в некотором смысле эквивалентен фрейму Уолша. Хорошо известно, что если $F=$ $\left[\mathbf{f}_{1}, \ldots, \mathbf{f}_{N}\right] \in \mathbb{C}^{m \times N}, m \leqslant N$, определяет фрейм Парсеваля для $\mathbb{C}^{m}$, причем $F F^{*}=I_{m}$ и $\left\|\mathbf{f}_{j}\right\|^{2}=\alpha$ для каждого $j \in\{1, \ldots, m\}$, то $\alpha=m / N$. Предположим, что матрица $F=\left[\mathbf{f}_{1}, \ldots, \mathbf{f}_{N}\right] \in \mathbb{C}^{m \times N}$ определяет такой фрейм Парсеваля для $\mathbb{C}^{m}$, что $\left\|\mathbf{f}_{j}\right\|=\sqrt{m / N}, j=1, \ldots, m$. Положим $G_{1}=F^{*}$, $G_{1}=\left[\mathbf{g}_{1}, \ldots, \mathbf{g}_{m}\right] \in \mathbb{C}^{N \times m}$, и дополним матрицу $G_{1}$ до ортогональной матрицы

$$
G=\left[G_{1} \mid G_{2}\right]=\left[\mathbf{g}_{1}, \ldots, \mathbf{g}_{m} \mid \mathbf{g}_{m+1}, \ldots, \mathbf{g}_{N}\right] \in \mathbb{C}^{N \times N},
$$

где $G_{2}=\left[\mathbf{g}_{m+1}, \ldots, \mathbf{g}_{N}\right] \in \mathbb{C}^{N \times(N-m)}$. Для матрицы $W=G^{*}$ имеем

$$
W=\left[\begin{array}{l}
F \\
\hline R
\end{array}\right]=\left[\begin{array}{llll}
\mathbf{f}_{1} & \mathbf{f}_{2} & \ldots & \mathbf{f}_{N} \\
\hline \mathbf{r}_{1} & \mathbf{r}_{2} & \ldots & \mathbf{r}_{N}
\end{array}\right]
$$

где $R=\left[\mathbf{r}_{1}, \ldots, \mathbf{r}_{N}\right] \in \mathbb{C}^{(N-m) \times N}$. Далее, полагая

$$
\mathbf{w}_{j}=\left[\begin{array}{c}
\mathbf{f}_{j} \\
\mathbf{r}_{j}
\end{array}\right], \quad j=1,2, \ldots, N
$$

будем иметь $W=\left[\mathbf{w}_{1}, \ldots, \mathbf{w}_{N}\right]$. Согласно предложению 3.1 система $\left\{\mathbf{w}_{1}, \ldots, \mathbf{w}_{N}\right\}$ является фреймом Парсеваля для $m$-мерного подпространства $S_{m} \subset \mathbb{C}^{N}$, состоящего из векторов вида

$$
\mathbf{z}=\left[\begin{array}{l}
\mathbf{x} \\
\mathbf{0}
\end{array}\right], \quad \mathbf{x} \in \mathbb{C}^{m}
$$

Фрейм $\left\{\mathbf{w}_{1}, \ldots, \mathbf{w}_{N}\right\}$ можно рассматривать как вложение исходного фрейма $\left\{\mathbf{f}_{1}, \ldots, \mathbf{f}_{N}\right\}$ в пространство $\mathbb{C}^{N}$. Пусть теперь $V=\left[\mathbf{v}_{1}, \ldots, \mathbf{v}_{N}\right]$ - матрица, совпадающая с нормализованной матрицей Уолша $H_{n} / \sqrt{N}$. Из определения видно, что матрица $V$ симметрична и ортогональна. Положим $P=V G$. Тогда $P W=V$ и, следовательно, $P \mathbf{w}_{j}=\mathbf{v}_{j}, j=1, \ldots, N$. Таким образом, каждому фрейму Парсеваля $\left\{\mathbf{f}_{1}, \ldots, \mathbf{f}_{N}\right\}$ для $\mathbb{C}^{m}$, определенному по векторам равной длины, поставлен в соответствие путем вложения в $\mathbb{C}^{N}$ фрейм Парсеваля $\left\{\mathbf{w}_{1}, \ldots, \mathbf{w}_{N}\right\}$, который ортогональным преобразованием переводится во фрейм Уолша. Изложенная в следующем разделе конструкция в случае $p=2$ представляет собой еще один метод построения фреймов Парсеваля с помощью матриц Уолша.

4. Фреймы Парсеваля и преобразование Виленкина-Крестенсона. Пусть $N=p^{n}$, где $p$ и $n$-натуральные числа, $p \geqslant 2$. Множество $\mathbb{Z}_{N}=\{0,1, \ldots, N-1\}$ является абелевой группой с операцией

$$
a \oplus b:=a \oplus_{p} b, \quad a, b \in \mathbb{Z}_{N}
$$

где $\oplus_{p}$ - операция сложения по модулю $p$, определяемая по разложениям

$$
a=\sum_{\nu=0}^{n-1} a_{\nu} p^{\nu}, \quad b=\sum_{\nu=0}^{n-1} b_{\nu} p^{\nu}, \quad a_{\nu}, b_{\nu} \in\{0,1, \ldots, p-1\} .
$$


(см. $[4, \S 1.5])$. Пространство $\ell^{2}\left(\mathbb{Z}^{N}\right)$ состоит из последовательностей вида

$$
x=(\ldots, x(-1), x(0), x(1), x(2), \ldots), \quad x(j) \in \mathbb{C}, \quad x(j+N)=x(j), \quad j \in \mathbb{Z} .
$$

Последовательность $x$ из $\ell^{2}\left(\mathbb{Z}^{N}\right)$ часто отождествляется с вектором $(x(0), x(1), \ldots, x(N-1))$. Линейные операции в пространстве $\ell^{2}\left(\mathbb{Z}^{N}\right)$ определяются покомпонентно. Скалярное произведение последовательностей $x, y \in \ell^{2}\left(\mathbb{Z}^{N}\right)$ определяется по формуле

$$
\langle x, y\rangle:=\sum_{j=0}^{N-1} x(j) \overline{y(j)} .
$$

Пусть $\varepsilon_{p}=\exp (2 \pi i / p)$. Функиии Крестенсона $w_{0}^{(N)}, w_{1}^{(N)}, \ldots, w_{N-1}^{(N)}$ для пространства $\ell^{2}\left(\mathbb{Z}^{N}\right)$ могут быть заданы равенствами

$$
w_{k}^{(N)}(l)=\varepsilon_{p}^{\sigma(k, l)}, \quad w_{k}^{(N)}(j)=w_{k}^{(N)}(j+N), \quad j \in \mathbb{Z},
$$

где

$$
\sigma(k, l)=\sum_{\nu=0}^{n-1} k_{\nu} l_{n-\nu-1}, \quad k=\sum_{\nu=0}^{n-1} k_{\nu} p^{\nu}, \quad l=\sum_{\nu=0}^{n-1} l_{\nu} p^{\nu}, \quad k_{\nu}, l_{\nu} \in\{0,1, \ldots, p-1\} .
$$

Из определений видно, что функции Крестенсона при $p=2$ совпадают с функциями Уолша. О свойствах дискретных функций Крестенсона и их применениях для обработки сигналов см. $[2$, $4,6]$.

Положим $N_{1}=p^{n-1}$. При $p=2$ для получения матрицы $\left(w_{k}^{(N)}(l)\right)$ следует каждую строку матрицы $\left(w_{k}^{\left(N_{1}\right)}(l)\right)$ написать дважды в виде двух новых строк и дополнить полученные строки, приписывая к первой строке справа еще один экземпляр этой же строки, а ко второй строке добавляя справа все элементы той же строки с противоположным знаком (см. раздел 2 и $[4, \S 1.3]$ ). Аналогично, при $p>2$ для получения матрицы $\left(w_{k}^{(N)}(l)\right)$ каждая строка матрицы $\left(w_{k}^{\left(N_{1}\right)}(l)\right)$ записывается $p$ раз в один столбец и умножается последовательно на $1, \varepsilon_{p}, \varepsilon_{p}^{2}, \ldots, \varepsilon_{p}^{p-1}$ (полученные после умножения строки приписываются справа к имеющимся строкам). При $p=3$ из матрицы

$$
\left(w_{k}^{(3)}(l)\right)=\left[\begin{array}{ccc}
1 & 1 & 1 \\
1 & \varepsilon & \varepsilon^{2} \\
1 & \varepsilon^{2} & \varepsilon
\end{array}\right], \quad k, l \in\{0,1,2\}
$$

этим методом получается следующая матрица (для краткости полагаем $\varepsilon=\varepsilon_{3}$ ):

$$
\left(w_{k}^{(9)}(l)\right)=\left[\begin{array}{ccccccccc}
1 & 1 & 1 & 1 & 1 & 1 & 1 & 1 & 1 \\
1 & 1 & 1 & \varepsilon & \varepsilon & \varepsilon & \varepsilon^{2} & \varepsilon^{2} & \varepsilon^{2} \\
1 & 1 & 1 & \varepsilon^{2} & \varepsilon^{2} & \varepsilon^{2} & \varepsilon & \varepsilon & \varepsilon \\
1 & \varepsilon & \varepsilon^{2} & 1 & \varepsilon & \varepsilon^{2} & 1 & \varepsilon & \varepsilon^{2} \\
1 & \varepsilon & \varepsilon^{2} & \varepsilon & \varepsilon^{2} & 1 & \varepsilon^{2} & \varepsilon & 1 \\
1 & \varepsilon & \varepsilon^{2} & \varepsilon^{2} & 1 & \varepsilon & \varepsilon & \varepsilon^{2} & 1 \\
1 & \varepsilon^{2} & \varepsilon & 1 & \varepsilon^{2} & \varepsilon & 1 & \varepsilon^{2} & \varepsilon \\
1 & \varepsilon^{2} & \varepsilon & \varepsilon & 1 & \varepsilon^{2} & \varepsilon^{2} & \varepsilon & 1 \\
1 & \varepsilon^{2} & \varepsilon & \varepsilon^{2} & \varepsilon & 1 & \varepsilon & 1 & \varepsilon^{2}
\end{array}\right], \quad k, l \in\{0,1, \ldots, 7\}
$$

Хорошо известно также, что

$$
\sum_{k=0}^{N-1} w_{k}^{(N)}(j)=\left\{\begin{array}{l}
N, \\
0, \text { если } j \text { не делится на } N .
\end{array} \quad \text { если } j \text { делится на } N,\right.
$$

Матрица $W^{(N)}:=\left(w_{l}^{(N)}(j)\right)_{l, j=0}^{N-1}$ симметрична и удовлетворяет соотношениям ортогональности

$$
\sum_{j=0}^{N-1} w_{l}^{(N)}(j) \overline{w_{k}^{(N)}(j)}=\sum_{i=0}^{N-1} w_{i}^{(N)}(l) \overline{w_{i}^{(N)}(k)}=N \delta_{l, k}, \quad l, k \in \mathbb{Z}_{N},
$$


где $\delta_{l, k}$ - символ Кронекера. Следовательно, система $\left\{w_{0}^{(N)}, w_{1}^{(N)}, \ldots, w_{N-1}^{(N)}\right\}$ является ортогональным базисом пространства $\ell^{2}\left(\mathbb{Z}^{N}\right)$.

Дискретное преобразование Виленкина-Кресенсона ставит в соответствие вектору $x$ из $\ell^{2}\left(\mathbb{Z}^{N}\right)$ последовательность $\widehat{x}$ коэффициентов Фурье вектора $x$ по системе $\left\{w_{0}^{(N)}, w_{1}^{(N)}, \ldots, w_{N-1}^{(N)}\right\}$ :

$$
\widehat{x}(k):=\frac{1}{N} \sum_{j=0}^{N-1} x(j) \overline{w_{k}^{(N)}(j)}, \quad k \in \mathbb{Z}_{N} .
$$

Разложение вектора $x$ по базису $\left\{w_{0}^{(N)}, w_{1}^{(N)}, \ldots, w_{N-1}^{(N)}\right\}$ записывается в виде

$$
x(j)=\sum_{k=0}^{N-1} \widehat{x}(k) w_{k}^{(N)}(j), \quad j \in \mathbb{Z}_{N} .
$$

Быстрые алгоритмы реализации прямого и обратного дискретного преобразования ВиленкинаКрестенсона содержатся в $[2,4,8]$.

Для каждого $k \in \mathbb{Z}_{N}$ оператор $p$-ичного сдвига $T_{k}: \ell^{2}\left(\mathbb{Z}^{N}\right) \rightarrow \ell^{2}\left(\mathbb{Z}^{N}\right)$ определяется по формуле

$$
\left(T_{k} x\right)(j):=x(j \ominus k), \quad x=x(j) \in \ell^{2}\left(\mathbb{Z}^{N}\right) .
$$

Для любых $x, y \in \ell^{2}\left(\mathbb{Z}^{N}\right), k, l \in \mathbb{Z}_{N}$ имеем

$$
\widehat{\left(T_{k} x\right)}(l)=\overline{w_{k}^{(N)}(l)} \widehat{x}(l), \quad\langle x, y\rangle=N\langle\widehat{x}, \widehat{y}\rangle .
$$

С помощью предложения 3.1 для любого $m \in\{N+1, \ldots, p N\}$ после выбора $m$ столбцов матрицы $(p N)^{-1 / 2} W^{(p N)}$ получаются фреймы Парсеваля из $m$ векторов для пространства $\ell^{2}\left(\mathbb{Z}^{N}\right)$. Следующая теорема в случае $p=2$ без доказательства приводилась в [10], а в ортогональном случае для любого $p \geqslant 2$ доказана в [13].

Теорема 4.1. Пусть векторы $u_{0}, u_{1}, \ldots, u_{r} \in \ell^{2}\left(\mathbb{Z}^{N}\right)$ таковы, что для матрицы

$$
M(l):=\frac{N}{\sqrt{p}}\left[\begin{array}{lll}
\widehat{u}_{0}(l) & \ldots & \widehat{u}_{r}(l) \\
\widehat{u}_{0}\left(l+N_{1}\right) & \ldots & \widehat{u}_{r}\left(l+N_{1}\right) \\
\widehat{u}_{0}\left(l+2 N_{1}\right) & \ldots & \widehat{u}_{r}\left(l+2 N_{1}\right) \\
\ldots & \ldots & \ldots \\
\widehat{u}_{0}\left(l+(p-1) N_{1}\right) & \ldots & \widehat{u}_{r}\left(l+(p-1) N_{1}\right)
\end{array}\right]
$$

при каждом $l=0,1, \ldots, N_{1}-1$ выполнено равенство

$$
M(l) M^{*}(l)=I_{p},
$$

где $I_{p}$ - единичная матрица порядка $р$. Тогда система

$$
B\left(u_{0}, u_{1}, \ldots, u_{r}\right):=\left\{T_{p k} u_{0}\right\}_{k=0}^{N_{1}-1} \cup\left\{T_{p k} u_{1}\right\}_{k=0}^{N_{1}-1} \cup \cdots \cup\left\{T_{p k} u_{r}\right\}_{k=0}^{N_{1}-1}
$$

является фреймом Парсеваля для $\ell^{2}\left(\mathbb{Z}^{N}\right)$.

Доказательство. По условию имеем

$$
\begin{gathered}
\sum_{s=0}^{r} \widehat{u}_{s}\left(l+\mu N_{1}\right) \overline{\widehat{u}_{s}\left(l+\nu N_{1}\right)}=0, \quad \mu \neq \nu, \\
\sum_{s=0}^{r}\left|\widehat{u}_{s}\left(l+\mu N_{1}\right)\right|^{2}=\frac{p}{N^{2}}, \quad \mu, \nu \in\{0,1, \ldots, p-1\} .
\end{gathered}
$$

Ввиду (5) достаточно убедиться, что для любого $x \in \mathbb{C}_{N}$ справедлива формула

$$
\|\widehat{x}\|^{2}=N \sum_{s=0}^{r} \sum_{k=0}^{N_{1}-1}\left|\left\langle\widehat{x}, \widehat{T_{p k} u_{s}}\right\rangle\right|^{2} .
$$


Для каждого $x \in \mathbb{C}_{N}$ согласно (5) в силу равенства $w_{p k}^{(N)}=w_{k}^{\left(N_{1}\right)}$ имеем

$$
\left|\left\langle\widehat{x}, \widehat{T_{p k} u_{s}}\right\rangle\right|^{2}=\left|\left\langle\widehat{x}, w_{p k}^{(N)} \widehat{u_{s}}\right\rangle\right|^{2}=\left|\sum_{l=0}^{N-1} \widehat{x}(l) w_{k}^{\left(N_{1}\right)}(l) \overline{u_{s}(l)}\right|^{2} .
$$

Полагая $a_{k}=\widehat{x}(l) w_{k}^{\left(N_{1}\right)}(l) \overline{\widehat{u_{s}}(l)}$ и применяя тождество

$$
\left|\sum_{l=0}^{N-1} a_{k}\right|^{2}=\sum_{l=0}^{N-1}\left|a_{k}\right|^{2}+2 \Re\left(a_{0} \sum_{l^{\prime}=1}^{N-1} \bar{a}_{l^{\prime}}+a_{1} \sum_{l^{\prime}=2}^{N-1} \bar{a}_{l^{\prime}}+\cdots+a_{N-2} \bar{a}_{N-1}\right),
$$

из (9) получим

$$
\left|\left\langle\widehat{x}, \widehat{T_{p k} u_{s}}\right\rangle\right|^{2}=\sum_{l=0}^{N-1}|\widehat{x}(l)|^{2}\left|\widehat{u_{s}}(l)\right|^{2}+2 \Re\left(A_{k}^{(N)}\left(\widehat{x}, \widehat{u_{s}}\right)\right),
$$

где

$$
\begin{aligned}
& A_{k}^{(N)}\left(\widehat{x}, \widehat{u_{s}}\right):=\widehat{x}(0) \overline{\hat{u}_{s}(0)} \sum_{l^{\prime}=1}^{N-1} \overline{w_{k}^{\left(N_{1}\right)}\left(l^{\prime}\right)} \widehat{x}\left(l^{\prime}\right) \widehat{u_{s}}\left(l^{\prime}\right)+ \\
& +\widehat{x}(1) \overline{\hat{u}_{s}(1)} \sum_{l^{\prime}=2}^{N-1} \overline{w_{k}^{\left(N_{1}\right)}\left(l^{\prime} \oplus 1\right)} \overline{\widehat{x}\left(l^{\prime}\right)} \widehat{u_{s}}\left(l^{\prime}\right)+ \\
& +\widehat{x}(2) \overline{\widehat{u}_{s}(2)} \sum_{l^{\prime}=3}^{N-1} \overline{w_{k}^{\left(N_{1}\right)}\left(l^{\prime} \oplus 2\right)} \overline{\widehat{x}\left(l^{\prime}\right)} \widehat{u_{s}}\left(l^{\prime}\right)+\cdots+ \\
& \left.+\widehat{x}(N-3) \overline{\widehat{u_{s}}(N-3)} \overline{\left(w_{k}^{\left(N_{1}\right)}(1)\right.} \overline{\widehat{x}(N-2)} \widehat{u_{s}}(N-2)+\overline{w_{k}^{\left(N_{1}\right)}(2)} \overline{\widehat{x}(N-1)} \widehat{u_{s}}(N-1)\right)+ \\
& +\widehat{x}(N-2) \overline{\widehat{u}_{s}(N-2)} \overline{w_{k}^{\left(N_{1}\right)}(1)} \overline{\widehat{x}(N-1)} \widehat{u}_{s}(N-1) .
\end{aligned}
$$

Применяя (7), имеем

$$
\begin{aligned}
& \sum_{s=0}^{r} \sum_{l=0}^{N-1}|\widehat{x}(l)|^{2}\left|\widehat{u_{s}}(l)\right|^{2}= \sum_{l=0}^{N-1}|\widehat{x}(l)|^{2} \sum_{s=0}^{r}\left|\widehat{u_{s}}(l)\right|^{2}= \\
&=\sum_{l=0}^{N_{1}-1}\left[\sum_{\mu=0}^{p-1}\left|\widehat{x}\left(l+\mu N_{1}\right)\right|^{2} \sum_{s=0}^{r}\left|\widehat{u_{s}}\left(l+\mu N_{1}\right)\right|^{2}\right]= \\
& \quad=\frac{p}{N^{2}} \sum_{l=0}^{N_{1}-1} \sum_{\mu=0}^{p-1}\left|\widehat{x}\left(l+\mu N_{1}\right)\right|^{2}=\frac{p}{N^{2}} \sum_{l=0}^{N-1}|\widehat{x}(l)|^{2} .
\end{aligned}
$$

Отсюда и из (10) получаем

$$
\sum_{s=0}^{r} \sum_{k=0}^{N_{1}-1}\left|\left\langle\widehat{x}, \widehat{T_{p k} u_{s}}\right\rangle\right|^{2}=\frac{p N_{1}}{N^{2}} \sum_{l=0}^{N-1}|\widehat{x}(l)|^{2}+2 \Re\left(\sum_{s=0}^{r} \sum_{k=0}^{N_{1}-1} A_{k}^{(N)}\left(\widehat{x}, \widehat{u_{s}}\right)\right) .
$$

Используя формулу (4) (с заменой $N_{1}$ на $N$ ), видим, что

$$
\begin{aligned}
\frac{1}{N_{1}} \sum_{k=0}^{N_{1}-1} A_{k}^{(N)}\left(\widehat{x}, \widehat{u_{s}}\right) & =\widehat{x}(0) \overline{\widehat{u_{s}}(0)} \overline{\widehat{x}\left(N_{1}\right)} \widehat{u_{s}}\left(N_{1}\right)+ \\
& +\widehat{x}(1) \overline{\widehat{u_{s}}(1)} \overline{\widehat{x}\left(N_{1}+1\right)} \widehat{u_{s}}\left(N_{1}+1\right)+\cdots+\widehat{x}\left(N_{1}-1\right) \overline{\widehat{u_{s}}\left(N_{1}-1\right)} \overline{\widehat{x}(N-1)} \widehat{u_{s}}(N-1)
\end{aligned}
$$

и, согласно (6),

$$
\sum_{s=0}^{r} \sum_{k=0}^{N_{1}-1} A_{k}^{(N)}\left(\widehat{x}, \widehat{u_{s}}\right)=0
$$


Замечая, что

$$
p N_{1}=N, \quad \sum_{l=0}^{N-1}|\widehat{x}(l)|^{2}=\|\widehat{x}\|^{2},
$$

из (11) и (12) получаем (8). Теорема 4.1 доказана.

Матрицу $M(l)$ из теоремы 4.1 для каждого $l=0,1, \ldots, N_{1}-1$ можно дополнить до унитарной матрицы. Поэтому для элементов матрицы $M(l)$ верны неравенства

$$
\left|\widehat{u}_{s}(l)\right|^{2}+\left|\widehat{u}_{s}\left(l+N_{1}\right)\right|^{2}+\cdots+\left|\widehat{u}_{s}\left(l+(p-1) N_{1}\right)\right|^{2} \leqslant \frac{p}{N^{2}},
$$

где $s=0,1, \ldots, r, l=0,1, \ldots, N_{1}-1$. Для случая, когда все неравенства в (13) обращаются в равенства, теорема 4.1 при $r=p-1$ соответствует ортонормированному базису $B\left(u_{0}, u_{1}, \ldots, u_{p-1}\right)$ в $\ell^{2}\left(\mathbb{Z}^{N}\right)$, построенному в [13].

В случае $r=N-1$ матрицу $M(l)$, удовлетворяющую условию $M(l) M^{*}(l)=I_{p}$, можно построить с помощью определенной выше матрицы $W^{(N)}$. Отметим, что при $p=2$ матрица $W^{(N)}$ совпадает с матрицей Уолша $W_{n}$ (см. раздел 2). Для иллюстрации случая $p=3$ приведем следующий пример.

Пример 4.1. Пусть $p=3, n=2, r=8$. Тогда

$$
M(l)=\frac{9}{\sqrt{3}}\left[\begin{array}{llll}
\widehat{u}_{0}(l) & \widehat{u}_{0}(l) & \ldots & \widehat{u}_{r}(l) \\
\widehat{u}_{0}(l+3) & \widehat{u}_{0}(l+3) & \ldots & \widehat{u}_{r}(l+3) \\
\widehat{u}_{0}(l+6) & \widehat{u}_{0}(l+6) & \ldots & \widehat{u}_{r}(l+6)
\end{array}\right], \quad l=0,1,2,
$$

и для выполнения условия $M(l) M^{*}(l)=I_{3}$ матрицы $M(0), M(1), M(2)$ можно выбрать так, чтобы составленная из них матрица $\left(\widehat{u}_{k}(j)\right)_{k, j=0}^{j}$ была пропорциональна матрице $\left(w_{k}^{(9)}(j)\right)_{k, j=0}^{8}$. Таким образом, в рассматриваемом случае матрица $M(l)$ формируется по строкам матрицы $W^{(9)}$.

Предположим, что $N$-мерный комплексный ненулевой вектор $\left(b_{0}, b_{1}, \ldots, b_{N-1}\right)$ удовлетворяет условию

$$
\left|b_{l}\right|^{2}+\left|b_{l+N_{1}}\right|^{2}+\cdots+\left|b_{l+(p-1) N_{1}}\right|^{2} \leqslant \frac{p}{N^{2}}, \quad l=0,1, \ldots, N_{1}-1
$$

Тогда по теореме 4.1 следующий алгоритм приводит к фрейму Парсеваля $B\left(u_{0}, u_{1}, \ldots, u_{r}\right)$ для $\ell^{2}\left(\mathbb{Z}^{N}\right)$.

\section{Алгоритм А.}

Шаг 1. Найти такой вектор $u_{0} \in \ell^{2}\left(\mathbb{Z}^{N}\right)$, что

$$
\widehat{u}_{0}(l)=b_{l}, \quad \widehat{u}_{0}\left(l+N_{1}\right)=b_{l+N_{1}}, \quad \ldots, \quad \widehat{u}_{0}\left(l+(p-1) N_{1}\right)=b_{l+(p-1) N_{1}}, \quad l=0,1, \ldots, N_{1}-1,
$$

где числа $b_{0}, b_{1}, \ldots, b_{N-1}$ берутся из (14).

Шаг 2. Для полученного на шаге 1 вектора $u_{0}$ найти такие векторы $u_{1}, \ldots, u_{r} \in \mathbb{C}_{N}$, что для матрицы

$$
M(l)=\frac{N}{\sqrt{p}}\left[\begin{array}{lll}
\widehat{u}_{0}(l) & \ldots & \widehat{u}_{r}(l) \\
\widehat{u}_{0}\left(l+N_{1}\right) & \ldots & \widehat{u}_{r}\left(l+N_{1}\right) \\
\widehat{u}_{0}\left(l+2 N_{1}\right) & \ldots & \widehat{u}_{r}\left(l+2 N_{1}\right) \\
\ldots & \ldots & \ldots \\
\widehat{u}_{0}\left(l+(p-1) N_{1}\right) & \ldots & \widehat{u}_{r}\left(l+(p-1) N_{1}\right)
\end{array}\right]
$$

при каждом $l=0,1, \ldots, N_{1}-1$ выполнено равенство $M(l) M^{*}(l)=I_{p}$.

Шаг 3. Определить систему $B\left(u_{0}, u_{1}, \ldots, u_{r}\right)$ по формуле

$$
B\left(u_{0}, u_{1}, \ldots, u_{r}\right)=\left\{T_{p k} u_{0}\right\}_{k=0}^{N_{1}-1} \cup\left\{T_{p k} u_{1}\right\}_{k=0}^{N_{1}-1} \cup \cdots \cup\left\{T_{p k} u_{r}\right\}_{k=0}^{N_{1}-1} .
$$

Шаг 1 этого алгоритма реализуется с помощью обратного дискретного преобразования Виленкина-Крестенсона:

$$
u_{0}(j)=\sum_{k=0}^{N-1} b_{k} w_{k}^{(N)}(j), \quad j \in \mathbb{Z}_{N}
$$


а для реализации шага 2 можно использовать те же методы, что и при построении фреймов на группах Виленкина (см. алгоритмы в $[22,23])$.

При обработке сигналов вектор $\left(b_{0}, b_{1}, \ldots, b_{N-1}\right)$ в алгоритме А может быть выбран по энтропийному, среднеквадратическому или иному критерию (cp. $[11,15])$.

В заключение отметим, что в дополнение к фрейму $B\left(u_{0}, u_{1}, \ldots, u_{r}\right)$ из теоремы 4.1 для каждого натурального $m, m \leqslant n$, аналогично ортогональному случаю (см. [13]) можно определить последовательность диадических фреймов Парсеваля $m$-го этапа для $\ell^{2}\left(\mathbb{Z}^{N}\right)$.

\section{СПИСОК ЛИТЕРАТУРЫ}

1. Агаев Г. Н., Виленкин Н. Я., Джафарли Г. М., Рубинштейн А. И. Мультипликативные системы функций и гармонический анализ на нульмерных группах. - Баку: Элм, 1981.

2. Беспалов М. С. Дискретное преобразование Крестенсона// Пробл. передачи информ. $-2010 .-46$, № 4. - C. 91-115.

3. Беспалов М. С. Собственные подпространства дискретного преобразования Уолша// Пробл. передачи информ. - 2010. - 46, № 3. - С. 60-79.

4. Голубов Б. И., Ефимов А. В., Скворцов В. А. Ряды и преобразования Уолша: Теория и применения. - М.: Изд-во ЛКИ, 2008.

5. Добеши И. Десять лекций по вейвлетам. - Ижевск: Регулярная и хаотическая динамика, 2001.

6. Залманзон Л. А. Преобразования Фурье, Уолша, Хаара и их применение в управлении, связи и других областях. - М.: Наука, 1989.

7. Малла C. Вейвлеты в обработке сигналов. - М.: Мир, 2005.

8. Малоземов В. Н. Машарский С. М. Основы дискретного гармонического анализа. - СПб.: Лань, 2012.

9. Новиков И. Я., Протасов В. Ю., Скопина М. А. Теория всплесков. - М.: Физматлит, 2006.

10. Робакидзе М. Г., Фарков Ю. А. Применение функций Уолша к построению фреймов Парсеваля в пространствах периодических последовательностей// в кн.: Современные проблемы теории функций и их приложения/ Мат. 19 Междунар. Саратов. зимней школы, посв. 90-летию акад. П. Л. Ульянова. - Саратов: Научная книга, 2018. - С. 265-267.

11. Родионов E. А. О применении вейвлетов к цифровой обработке сигналов// Изв. Саратов. ун-та. Нов. сер. Сер. Мат. Мех. Информ. - 2016. - 16, № 2. - С. 217-225.

12. Рябцов И. С. Необходимые и достаточные условия простоты фреймов Парсеваля// Вестн. СамГУ. Естественнонауч. сер. - 2012. - № 6 (97). - С. 42-48.

13. Фарков Ю. А. Дискретные вейвлеты и преобразование Виленкина-Крестенсона// Мат. заметки. 2011. - 89, № 6. - C. 914-928.

14. Фарков Ю. А. Ортогональные всплески в анализе Уолша// в кн.: Современные проблемы математики и механики (Лукашенко Т. П., Солодова А. П., ред.)/ K 80-летию В. А. Скворцова. Обобщенные интегралы и гармонический анализ. - М.: Изд-во Московского университета, 2016. - Т. 11. - С. $62-$ 75 .

15. Фарков Ю. А. Параметрические множества для фреймов в анализе Уолша// Вестн. Евраз. нац. ун-та им. Л. Н. Гумилева. Сер. Мат. Информ. Мех. - 2018. - 124, № 3. - С. 89-94.

16. Albrecht A., Howlett P., Verma G. Optimal splitting of Parseval frames using Walsh matrices// Poincaré J. Anal. Appl. — 2018. — № 2. - P. 39-58.

17. Balonin N. A., Doković D. Ž., Karbovskiy D. A. Construction of symmetric Hadamard matrices of order $4 \nu$ for $\nu=47,73,113 / /$ Spec. Matrices. - 2018. - 6. - P. 11-22.

18. Bownik M. The Kadison-Singer problem// in: Frames and Harmonic Analysis. - Providence, Rhode Island: Am. Math. Soc., 2018. - P. 63-92.

19. Casazza P. G.,Tremain J. C. The Kadison-Singer problem in mathematics and engineering// Proc. Natl. Acad. Sci. USA. — 2006. - 103, № 7. — P. 2032-2039.

20. Christensen O. An Inroduction to Frames and Riesz Bases. - Boston: Birkhäuser, 2016.

21. Farkov Yu. A. Examples of frames on the Cantor dyadic group// J. Math. Sci. - 2012. — 187, № 1. P. 22-34.

22. Farkov Yu. A. Constructions of MRA-based wavelets and frames in Walsh analysis// Poincaré J. Anal. Appl. — 2015. - № 2. - P. 13-36.

23. Farkov Yu. A., Lebedeva E. A., Skopina M. A. Wavelet frames on Vilenkin groups and their approximation properties// Int. J. Wavelets Multires. Inf. Process - 2015. — 13, № 5. - 1550036 . 
24. Farkov Yu. A. Wavelet frames related to Walsh functions// Eur. J. Math. - 2019. - 5. - P. $250-267$.

25. Farkov Yu. A., Manchanda P., Siddiqi A. H. Construction of Wavelets through Walsh Functions. - Singapore: Springer, 2019.

26. Hedayat A., Wallis W. D. Hadamard matrices and their applications// Ann. Stat. — 1978. — 6, № 6. P. 1184-1238.

27. Kadison R. V., Singer I. M. Extensions of pure states// Am. J. Math. - 1959. - 81. - P. $383-400$.

28. Krivoshein A., Protasov V., Skopina M. Multivariate Wavelet Frames. - Singapore: Springer, 2016.

29. Lang W. C. Orthogonal wavelets on the Cantor dyadic group// SIAM J. Math. Anal. — 1996. — 27 , № 1 . - P. 305-312.

30. Schipp F., Wade W. R., Simon P. Walsh Series: An Introduction to Dyadic Harmonic Analysis. — New York: Adam Hilger, 1990.

31. Seberry J., Wysocki B. J., Wysocki T. A. On some applications of Hadamard matrices// Metrika. — 2005. - 62, № 2. - P. 221-239.

32. Dyadic Walsh analysis from 1924 onwards Walsh-Gibbs-Butzer dyadic differentiation in science. — Amsterdam: Atlantis Press, 2015.

33. Stevens M. The Kadison-Singer Property. - Berlin: Springer, 2016.

34. Waldron S. An Introduction to Finite Tight Frames. — New York: Birkhäuser, 2018.

35. Weaver N. The Kadison-Singer problem in discrepancy theory// Discrete Math. — 2004. — 278, № 1-3. - P. 227-239.

Фарков Юрий Анатольевич

Российская академия народного хозяйства и государственной службы при Президенте РФ

E-mail: farkov@list.ru, farkov-ya@ranepa.ru 